\title{
ZnO based Quantum Dots for Magnetic Resonance and Fluorescence Imaging
}

\author{
Leila A. Chiavacci ${ }^{1,2}$, Bruna Lallo da Silva ${ }^{1,2}$, Eloisa Berbel Manaia ${ }^{3}$, Elise Lepeltier ${ }^{2}$, \\ Jean-Pierre Benoit ${ }^{2}$, Laurent Lemaire ${ }^{2}$ \\ ${ }^{1}$ Department of Drugs and Medicines, School of Pharmaceutical Sciences, São Paulo State University (UNESP) \\ 14800-903, Araraquara, SP, Brazil \\ leila.chiavacci@unesp.br; bruna.lallo@unesp.br \\ ${ }^{2}$ MINT, INSERM U1066- CNRS 6021- Université Angers, CHU -IBS \\ 4 rue Larrey, 49933 Angers, France \\ laurent.lemaire@univ-angers.fr; elise.lepeltier@univ-angers.fr; jean-pierre.benoit@univ-angers.fr \\ ${ }^{3}$ Institut Galien Paris-Sud, Univ. Paris-Sud, CNRS, Université Paris-Saclay \\ 92290 Châtenay-Malabry, France \\ eloisa.berbel-manaia@u-psud.fr
}

\section{Extended Abstract}

Theranostic nanocarriers combine a diagnostic and therapy agent allowing both imaging and treatment. Usually, the diagnostic agents used in theranostics are fluorescent dyes or quantum dots (QDs). In recent years, efforts have been made to develop new nanoprobes to associate magnetic resonance imaging (MRI) to fluorescence imaging (FI) involving QDs containing Gd. The ZnO-based QDs are a good candidate for imaging agent due to their excellent photoluminescence properties and low toxicity; however, studies have shown that $\mathrm{ZnO}$ QDs degrade rapidly in biological acid medium. To overcome this limitation of biological use in this work we adopt two strategies: in the first one we have incorpored Gd-doped $\mathrm{ZnO}$ QDs in lipidic nanocapsules aiming to protect the QDs of biological degradation. In the second one we have developed and characterized gadolinium-copper-indium-sulfur (GCIS) and ZnS (core/shell) conjugated to ZnO (GCIS/ZnS/ZnO QDs) that exhibit pronounced near-infrared fluorescence and good colloidal stability in different $\mathrm{pH}$ ranges [1]. Both Gd-doped $\mathrm{ZnO}$ QDs and GCIS/ZnS/ZnO QDs were characterized by XRD and by photoluminescence spectroscopy to evaluate their structure and optical properties. The results showed that the peaks in the XRD of Gd-doped ZnO QDs correspond to ZnO wurtzite hexagonal phase without any formation of $\mathrm{Gd}_{2} \mathrm{O}_{3}$ oxide phase, whatever the concentration of $\mathrm{Gd}^{3+}$ ions. The XRD profiles of GCIS/ZnS/ZnO QDs showed the same structure of ZnO and the peaks of the GCIS/ZnS QDs were not detected due to their lower proportion in relation to $\mathrm{ZnO}$. PL spectra of Gd-doped $\mathrm{ZnO}$ QDs showed a shift towards the low excitation/emission wavelengths with the increase of Gd content; moreover, a decrease in PL intensity was observed with Gd content. We have investigated the internalization of Gd-doped ZnO QDs into lipidic nanocapsules by J774 murine macrophage-like cells and A549 human lung cancer cells using fluorescence microscopy. Thanks to their visible emission we could shed light their different localization within the cells as a function of incubation time. As expected, PL spectra of GCIS/ZnS/ZnO QDs showed emission in the near infrared region (NIR), differently from Gd-doped ZnO QDs (yellow emission). The results of stability of the systems in biological medium showed that the incorporation of the quantum dots in lipidic nanocapsules increases the $\mathrm{ZnO}$ based quantum dots stability.

\section{References}

[1] W. Yang, W. Guo, X. Gong, B. Zhang, S. Wang, N. Chen, W. Yang, Y. Tu, X. Fang, J. Chang, "Facile Synthesis of Gd-Cu-In-S/ZnS Bimodal Quantum Dots with Optimized Properties for Tumor Targeted Fluorescence/MR In VivoImaging," ACS Apllied Materials \& Interfaces, vol. 7, no. 33, pp. 18759-18768, 2015. 